\title{
Some new identities of Frobenius-Euler numbers and polynomials
}

Dae San Kim ${ }^{1}$ and Taekyun Kim²

"Correspondence: tkkim@kw.ac.kr ${ }^{2}$ Department of Mathematics, Kwangwoon University, Seoul, 139-701, Republic of Korea

Full list of author information is available at the end of the article

\section{Abstract}

In this paper, we give some new and interesting identities which are derived from the basis of Frobenius-Euler. Recently, several authors have studied some identities of Frobenius-Euler polynomials. From the methods of our paper, we can also derive many interesting identities of Frobenius-Euler numbers and polynomials.

\section{Introduction}

Let $\lambda(\neq 1) \in \mathbf{C}$. As is well known, the Frobienius-Euler polynomials are defined by the generating function to be

$$
\frac{1-\lambda}{e^{t}-\lambda} e^{x t}=e^{H(x \mid \lambda) t}=\sum_{n=0}^{\infty} H_{n}(x \mid \lambda) \frac{t^{n}}{n !}
$$

with the usual convention about replacing $H^{n}(x \mid \lambda)$ by $H_{n}(x \mid \lambda)$ (see [1-6]).

In the special case, $x=0, H_{n}(0 \mid \lambda)=H_{n}(\lambda)$ are called the $n$th Frobenius-Euler numbers. Thus, by (1), we get

$$
(H(\lambda)+1)^{n}-\lambda H_{n}(\lambda)=H_{n}(1 \mid \lambda)-\lambda H_{n}(\lambda)=(1-\lambda) \delta_{0, n},
$$

where $\delta_{0, n}$ is the Kronecker symbol.

From (1), we can derive the following equation:

$$
H_{n}(x \mid \lambda)=(H(\lambda)+x)^{n}=\sum_{0 \leq l \leq n}\left(\begin{array}{l}
n \\
l
\end{array}\right) H_{n-l}(\lambda) x^{l} \quad(\text { see }[6-16]) .
$$

Thus, by (3), we easily see that the leading coefficient of $H_{n}(x \mid \lambda)$ is $H_{0}(\lambda)=1$. So, $H_{n}(x \mid \lambda)$ are monic polynomials of degree $n$ with coefficients in $\mathbf{Q}(\lambda)$.

From (1), we have

$$
\sum_{n=0}^{\infty}\left(H_{n}(x+1 \mid \lambda)-\lambda H_{n}(x \mid \lambda)\right) \frac{t^{n}}{n !}=\frac{(1-\lambda) e^{(x+1) t}}{e^{t}-\lambda}-\lambda \frac{1-\lambda}{e^{t}-\lambda} e^{x t} .
$$

Thus, by (4), we get

$$
H_{n}(x+1 \mid \lambda)-\lambda H_{n}(x \mid \lambda)=(1-\lambda) x^{n}, \quad \text { for } n \in \mathbf{Z}_{+} .
$$

(0) 2012 Kim and Kim; licensee Springer. This is an Open Access article distributed under the terms of the Creative Commons Attribution License (http://creativecommons.org/licenses/by/2.0), which permits unrestricted use, distribution, and reproduction in any medium, provided the original work is properly cited. 
It is easy to show that

$$
\frac{d}{d x} H_{n}(x \mid \lambda)=\frac{d}{d x}(H(\lambda)+x)^{n}=n H_{n-1}(x \mid \lambda) \quad(n \in \mathbf{N}) .
$$

From (6), we have

$$
\int_{0}^{1} H_{n}(x \mid \lambda) d x=\frac{1}{n+1}\left(H_{n+1}(1 \mid \lambda)-H_{n+1}(\lambda)\right)=\frac{\lambda-1}{n+1} H_{n+1}(\lambda) .
$$

Let $\mathbb{P}_{n}(\lambda)=\{p(x) \in \mathbf{Q}(\lambda)[x] \mid \operatorname{deg} p(x) \leq n\}$ be a vector space over $\mathbf{Q}(\lambda)$. Then we note that $\left\{H_{0}(x \mid \lambda), H_{1}(x \mid \lambda), \ldots, H_{n}(x \mid \lambda)\right\}$ is a good basis for $\mathbb{P}_{n}(\lambda)$.

In this paper, we develop some new methods to obtain some new identities and properties of Frobenius-Euler polynomials which are derived from the basis of Frobenius-Euler polynomials. Those methods are useful in studying the identities of Frobenius-Euler polynomials.

\section{Some identities of Frobenius-Euler polynomials}

Let us take $p(x) \in \mathbb{P}_{n}(\lambda)$. Then $p(x)$ can be expressed as a $\mathbf{Q}(\lambda)$-linear combination of $H_{0}(x \mid \lambda), \ldots, H_{n}(x \mid \lambda)$ as follows:

$$
p(x)=b_{0} H_{0}(x \mid \lambda)+b_{1} H_{1}(x \mid \lambda)+\cdots+b_{n} H_{n}(x \mid \lambda)=\sum_{0 \leq k \leq n} b_{k} H_{k}(x \mid \lambda) .
$$

Let us define the operator $\Delta_{\lambda}$ by

$$
g(x)=\triangle_{\lambda} p(x)=p(x+1)-\lambda p(x) .
$$

From (9), we can derive the following equation (10):

$$
g(x)=\triangle_{\lambda} p(x)=\sum_{0 \leq k \leq n} b_{k}\left(H_{k}(x+1 \mid \lambda)-\lambda H_{k}(x \mid \lambda)\right)=(1-\lambda) \sum_{0 \leq k \leq n} b_{k} x^{k} .
$$

For $r \in \mathbf{Z}_{+}$, let us take the $r$ th derivative of $g(x)$ in (10) as follows:

$$
g^{(r)}(x)=(1-\lambda) \sum_{r \leq k \leq n} k(k-1) \cdots(k-r+1) b_{k} x^{k-r}, \quad \text { where } g^{(r)}(x)=\frac{d^{r} g(x)}{d x^{r}}
$$

Thus, by (11), we get

$$
g^{r}(0)=\left.\frac{d^{r} g(x)}{d x^{r}}\right|_{x=0}=(1-\lambda) r ! b_{r}
$$

From (12), we have

$$
b_{r}=\frac{g^{(r)}(0)}{(1-\lambda) r !}=\frac{1}{(1-\lambda) r !}\left(p^{(r)}(1)-\lambda p^{(r)}(0)\right)
$$

where $r \in \mathbf{Z}_{+}$and $p^{(r)}(0)=\left.\frac{d^{r} p(x)}{d x^{r}}\right|_{x=0}$. Therefore, by (13), we obtain the following theorem. 
Theorem 1 For $\lambda(\neq 1) \in \mathbf{C}, n \in \mathbf{Z}_{+}$, let $p(x) \in \mathbb{P}_{n}(\lambda)$ with $p(x)=\sum_{0 \leq k \leq n} b_{k} H_{k}(x \mid \lambda)$. Then we have

$$
b_{k}=\frac{1}{(1-\lambda) k !} g^{(k)}(0)=\frac{1}{(1-\lambda) k !}\left(p^{(k)}(1)-\lambda p^{(k)}(0)\right) .
$$

Let us take $p(x)=H_{n}\left(x \mid \lambda^{-1}\right)$. Then, by Theorem 1 , we get

$$
H_{n}\left(x \mid \lambda^{-1}\right)=\sum_{0 \leq k \leq n} b_{k} H_{k}(x \mid \lambda)
$$

where

$$
\begin{aligned}
b_{k} & =\frac{1}{(1-\lambda) k !} \frac{n !}{(n-k) !}\left\{H_{n-k}\left(1 \mid \lambda^{-1}\right)-\lambda H_{n-k}\left(\lambda^{-1}\right)\right\} \\
& =\frac{1}{1-\lambda}\left(\begin{array}{l}
n \\
k
\end{array}\right)\left\{H_{n-k}\left(1 \mid \lambda^{-1}\right)-\lambda H_{n-k}\left(\lambda^{-1}\right)\right\} \\
& =\frac{1}{1-\lambda}\left(\begin{array}{l}
n \\
k
\end{array}\right)\left\{\left(1-\lambda^{-1}\right) 0^{n-k}+\frac{1}{\lambda} H_{n-k}\left(\lambda^{-1}\right)-\lambda H_{n-k}\left(\lambda^{-1}\right)\right\} .
\end{aligned}
$$

By (14) and (15), we get

$$
\begin{aligned}
& H_{n}\left(x \mid \lambda^{-1}\right) \\
& =-\frac{1}{\lambda} H_{n}(x \mid \lambda)+\sum_{k=0}^{n}\left\{\frac{\left(\begin{array}{l}
n \\
k
\end{array}\right)}{\lambda(1-\lambda)} H_{n-k}\left(\lambda^{-1}\right)-\frac{\lambda\left(\begin{array}{l}
n \\
k
\end{array}\right)}{1-\lambda} H_{n-k}\left(\lambda^{-1}\right)\right\} H_{k}(x \mid \lambda) \\
& =-\frac{1}{\lambda} H_{n}(x \mid \lambda)+\sum_{k=0}^{n}\left(\begin{array}{l}
n \\
k
\end{array}\right) \frac{1+\lambda}{\lambda} H_{n-k}\left(\lambda^{-1}\right) H_{k}(x \mid \lambda) .
\end{aligned}
$$

Therefore, by (16), we obtain the following theorem.

Theorem 2 For $n \in \mathbf{Z}_{+}$, we have

$$
\lambda H_{n}\left(x \mid \lambda^{-1}\right)+H_{n}(x \mid \lambda)=(1+\lambda) \sum_{0 \leq k \leq n}\left(\begin{array}{l}
n \\
k
\end{array}\right) H_{n-k}\left(\lambda^{-1}\right) H_{k}(x \mid \lambda) .
$$

Let

$$
p(x)=\sum_{0 \leq k \leq n} H_{k}(x \mid \lambda) H_{n-k}(x \mid \lambda) \in \mathbb{P}_{n}(\lambda) .
$$

From Theorem 2, we note that $p(x)$ can be generated by $\left\{H_{0}(x \mid \lambda), H_{1}(x \mid \lambda), \ldots, H_{n}(x \mid \lambda)\right\}$ as follows:

$$
p(x)=\sum_{0 \leq k \leq n} H_{k}(x \mid \lambda) H_{n-k}(x \mid \lambda)=\sum_{0 \leq k \leq n} b_{k} H_{k}(x \mid \lambda) .
$$

By (17), we get

$$
p^{(k)}(x)=\frac{(n+1) !}{(n-k+1) !} \sum_{k \leq l \leq n} H_{l-k}(x \mid \lambda) H_{n-k}(x \mid \lambda),
$$


and

$$
\begin{aligned}
b_{k}= & \frac{1}{(1-\lambda) k !}\left\{p^{(k)}(1)-\lambda p^{(k)}(0)\right\} \\
= & \frac{(n+1) !}{(1-\lambda) k !(n-k+1) !} \sum_{l=k}^{n}\left\{H_{l-k}(1 \mid \lambda) H_{n-l}(1 \mid \lambda)-\lambda H_{l-k}(\lambda) H_{n-l}(\lambda)\right\} \\
= & \frac{n+1}{(1-\lambda)(n-k+1)}\left(\begin{array}{l}
n \\
k
\end{array}\right) \sum_{l=k}^{n}\left\{\left(\lambda H_{l-k}(\lambda)+(1-\lambda) \delta_{0, l-k}\right)\left(\lambda H_{n-l}+(1-\lambda) \delta_{0, n-l}\right)\right. \\
& \left.-\lambda H_{l-k}(\lambda) H_{n-l}(\lambda)\right\} \\
= & \frac{n+1}{(1-\lambda)(n-k+1)}\left(\begin{array}{l}
n \\
k
\end{array}\right) \sum_{l=k}^{n}\left\{\lambda(1-\lambda) \delta_{0, l-k} H_{n-l}(\lambda)+\lambda(1-\lambda)\right. \\
& \left.\times H_{l-k}(\lambda) \delta_{0, n-l}+(1-\lambda)^{2} \delta_{0, l-k} \delta_{0, n-l}+\lambda(\lambda-1) H_{l-k}(\lambda) H_{n-l}(\lambda)\right\} \\
= & \frac{n+1}{(1-\lambda)(n-k+1)}\left(\begin{array}{l}
n \\
k
\end{array}\right) \sum_{l=k}^{n}\left\{\lambda(\lambda-1) H_{l-k}(\lambda) H_{n-l}(\lambda)+\lambda(1-\lambda)\right. \\
& \left.\times H_{n-k}(\lambda)+\lambda(1-\lambda) H_{n-k}(\lambda)+(1-\lambda)^{2} \delta_{n, k}\right\} \\
= & \frac{n+1}{n-k+1}\left(\begin{array}{l}
n \\
k
\end{array}\right) \sum_{l=k}^{n}\left\{-\lambda H_{l-k}(\lambda) H_{n-l}(\lambda)+2 \lambda H_{n-k}(\lambda)+(1-\lambda) \delta_{n, k}\right\} .
\end{aligned}
$$

From (18) and (20), we have

$$
\begin{aligned}
\sum_{0 \leq k \leq n} H_{k}(x \mid \lambda) H_{n-k}(x \mid \lambda)= & (n+1) \sum_{0 \leq k \leq n-1} \frac{\left(\begin{array}{l}
n \\
k
\end{array}\right)}{n-k+1} \sum_{k \leq l \leq n}\left\{(-\lambda) H_{l-k}(\lambda) H_{n-l}(\lambda)\right. \\
& \left.+2 \lambda H_{n-k}(\lambda)\right\} H_{k}(x \mid \lambda)+(n+1) H_{n}(x \mid \lambda) .
\end{aligned}
$$

Therefore, by (21), we obtain the following theorem.

Theorem 3 For $n \in \mathbf{Z}_{+}$, we have

$$
\begin{aligned}
& \frac{1}{n+1} \sum_{0 \leq k \leq n} H_{k}(x \mid \lambda) H_{n-k}(x \mid \lambda) \\
& =\sum_{0 \leq k \leq n-1} \frac{\left(\begin{array}{l}
n \\
k
\end{array}\right)}{n-k+1} \sum_{k \leq l \leq n}\left\{(-\lambda) H_{l-k}(\lambda) H_{n-l}(\lambda)+2 \lambda H_{n-k}(\lambda)\right\} H_{k}(x \mid \lambda)+H_{n}(x \mid \lambda) .
\end{aligned}
$$

Let us consider

$$
p(x)=\sum_{k=0}^{n} \frac{1}{k !(n-k) !} H_{k}(x \mid \lambda) H_{n-k}(x \mid \lambda) \in \mathbb{P}_{n}(\lambda) .
$$

By Theorem $1, p(x)$ can be expressed by

$$
p(x)=\sum_{k=0}^{n} b_{k} H_{k}(x \mid \lambda)
$$


From (22), we have

$$
p^{(r)}(x)=2^{r} \sum_{k=r}^{n} \frac{H_{k-r}(x \mid \lambda) H_{n-k}(x \mid \lambda)}{(k-r) !(n-k) !} \quad\left(r \in \mathbf{Z}_{+}\right) .
$$

By Theorem 1, we get

$$
\begin{aligned}
b_{k}= & \frac{1}{2 k !}\left\{p^{(k)}(1)-p^{(k)}(0)\right\} \\
= & \frac{2^{k-1}}{k !} \sum_{l=k}^{n} \frac{1}{(l-k) !(n-l) !}\left\{H_{l-k}(1 \mid \lambda) H_{n-l}(1 \mid \lambda)-\lambda H_{l-k}(\lambda) H_{n-l}(\lambda)\right\} \\
= & \frac{2^{k-1}}{k !} \sum_{l=k}^{n} \frac{1}{(l-k) !(n-l) !}\left\{\left(\lambda H_{l-k}(\lambda)+(1-\lambda) \delta_{0, l-k}\right)\left(\lambda H_{n-l}(\lambda)+(1-\lambda) \delta_{0, n-l}\right)\right. \\
& \left.-\lambda H_{l-k}(\lambda) H_{n-l}(\lambda)\right\} \\
= & \frac{2^{k-1}}{k !}\left\{\sum_{l=k}^{n} \frac{\lambda(\lambda-1) H_{l-k}(\lambda) H_{n-l}(\lambda)}{(l-k) !(n-l) !}+\frac{2 \lambda(1-\lambda) H_{n-k}(\lambda)}{(n-k) !}+(1-\lambda)^{2} \delta_{n, k}\right\} \\
= & \begin{cases}\frac{2^{k-1}}{k !} \sum_{l=k}^{n}\left\{\frac{\lambda(\lambda-1) H_{l-k}(\lambda) H_{n-l}(\lambda)}{(l-k) !(n-l) !}+\frac{2 \lambda(1-\lambda) H_{n-k}(\lambda)}{(n-k) !}\right\}, & \text { if } k \neq n, \\
\frac{2^{n-1}(1-\lambda)}{n !}, & \text { if } k=n .\end{cases}
\end{aligned}
$$

Therefore, by (25), we obtain the following theorem.

Theorem 4 For $n \in \mathbf{Z}_{+}$, we have

$$
\begin{aligned}
& \sum_{0 \leq k \leq n} \frac{1}{k !(n-k) !} H_{k}(x \mid \lambda) H_{n-k}(x \mid \lambda) \\
& =\sum_{0 \leq k \leq n-1} \frac{2^{k-1}}{k !} \sum_{k \leq l \leq n}\left\{\frac{\lambda(\lambda-1) H_{l-k}(\lambda) H_{n-l}(\lambda)}{(l-k) !(n-l) !}+\frac{2 \lambda(1-\lambda) H_{n-k}(\lambda)}{(n-k) !}\right\} H_{k}(x \mid \lambda) \\
& \quad+\frac{2^{n-1}(1-\lambda)}{n !} H_{n}(x \mid \lambda) .
\end{aligned}
$$

\section{Higher-order Frobenius-Euler polynomials}

For $n \in \mathbf{Z}_{+}$, the Frobenius-Euler polynomials of order $r$ are defined by the generating function to be

$$
\begin{aligned}
\left(\frac{1-\lambda}{e^{t}-\lambda}\right)^{r} e^{x t} & =e^{H^{(r)}(x \mid \lambda) t} \\
& =\sum_{n=0}^{\infty} H_{n}^{(r)}(x \mid \lambda) \frac{t^{n}}{n !},
\end{aligned}
$$

with the usual convention about replacing $\left(H^{(r)}(x \mid \lambda)\right)^{n}$ by $H_{n}^{(r)}(x \mid \lambda)$ (see [1-10]). In the special case, $x=0, H_{n}^{(r)}(0 \mid \lambda)=H_{n}^{(r)}(\lambda)$ are called the $n$th Frobenius-Euler numbers of order $r$ (see $[8,9])$. 
From (26), we have

$$
H_{n}^{(r)}(x \mid \lambda)=\left(H^{(r)}(\lambda)+x\right)^{n}=\sum_{l=0}^{n}\left(\begin{array}{l}
n \\
l
\end{array}\right) H_{n-l}^{(r)}(\lambda) x^{l}
$$

with the usual convention about replacing $\left(H^{(r)}(\lambda)\right)^{n}$ by $H_{n}^{(r)}(\lambda)$.

By (26), we get

$$
H_{n}^{(r)}(\lambda)=\sum_{n_{1}+\cdots+n_{r}=n}\left(\begin{array}{c}
n \\
n_{1}, n_{2}, \ldots, n_{r}
\end{array}\right) H_{n_{1}}(\lambda) \cdots H_{n_{r}}(\lambda),
$$

where $\left(\begin{array}{c}n \\ n_{1}, n_{2}, \ldots, n_{r}\end{array}\right)=\frac{n !}{n_{1} ! n_{2} ! \cdots n_{r} !}$. From (27) and (28), we note that the leading coefficient of $H_{n}^{(r)}(x \mid \lambda)$ is given by

$$
\begin{aligned}
H_{0}^{(r)}(\lambda) & =\sum_{n_{1}+\cdots+n_{r}=0}\left(\begin{array}{c}
n \\
n_{1}, n_{2}, \ldots, n_{r}
\end{array}\right) H_{n_{1}}(\lambda) \cdots H_{n_{r}}(\lambda) \\
& =H_{0}(\lambda) \cdots H_{0}(\lambda)=1 .
\end{aligned}
$$

Thus, by (29), we see that $H_{n}^{(r)}$ is a monic polynomial of degree $n$ with coefficients in $\mathbf{Q}(\lambda)$. From (26), we have

$$
H_{n}^{(0)}(x \mid \lambda)=x^{n}, \quad \text { for } n \in \mathbf{Z}_{+},
$$

and

$$
\frac{\partial}{\partial x} H_{n}^{(r)}(x \mid \lambda)=\frac{\partial}{\partial x}\left(H^{(r)}(\lambda)+x\right)^{n}=n H_{n-1}^{(r)}(x \mid \lambda) \quad(r \geq 0) .
$$

It is not difficult to show that

$$
H_{n}^{(r)}(x+1 \mid \lambda)-\lambda H_{n}^{(r)}(x \mid \lambda)=(1-\lambda) H_{n}^{(r-1)}(x \mid \lambda) .
$$

Now, we note that $\left\{H_{0}^{(r)}(x \mid \lambda), H_{1}^{(r)}(x \mid \lambda), \ldots, H_{n}^{(r)}(x \mid \lambda)\right\}$ is also a good basis for $\mathbb{P}_{n}(\lambda)$.

Let us define the operator $D$ as $D f(x)=\frac{d f(x)}{d x}$ and let $p(x) \in \mathbb{P}_{n}(\lambda)$. Then $p(x)$ can be written as

$$
p(x)=\sum_{k=0}^{n} C_{k} H_{k}^{(r)}(x \mid \lambda)
$$

From (9) and (32), we have

$$
\triangle_{\lambda} H_{n}^{(r)}(x \mid \lambda)=H_{n}^{(r)}(x+1 \mid \lambda)-\lambda H_{n}^{(r)}(x \mid \lambda)=(1-\lambda) H_{n}^{(r-1)}(x \mid \lambda) .
$$

Thus, by (33) and (34), we get

$$
\triangle_{\lambda}^{r} p(x)=(1-\lambda)^{r} \sum_{k=0}^{n} C_{k} H_{k}^{(0)}(x \mid \lambda)=(1-\lambda)^{r} \sum_{k=0}^{n} C_{k} x^{k}
$$

Let us take the $k$ th derivative of $\triangle_{\lambda}^{r} p(x)$ in (35). 
Then we have

$$
D^{k}\left(\triangle_{\lambda}^{r} p(x)\right)=(1-\lambda)^{r} \sum_{l=k}^{n} \frac{l !}{(l-k) !} C_{l} x^{l-k}
$$

Thus, from (36), we have

$$
D^{k}\left(\triangle_{\lambda}^{r} p(0)\right)=(1-\lambda)^{r} \sum_{l=k}^{n} \frac{l ! C_{l}}{(l-k) !} 0^{l-k}=(1-\lambda)^{r} k ! C_{k} .
$$

Thus, by (37), we get

$$
\begin{aligned}
C_{k} & =\frac{D^{k}\left(\triangle_{\lambda}^{r} p(0)\right)}{(1-\lambda)^{r} k !} \\
& =\frac{\triangle_{\lambda}^{r}\left(D^{k} p(0)\right)}{(1-\lambda)^{r} k !}=\frac{1}{(1-\lambda)^{r} k !} \sum_{j=0}^{r}\left(\begin{array}{l}
r \\
j
\end{array}\right)(-\lambda)^{(r-j)} D^{k} p(j) .
\end{aligned}
$$

Therefore, by (33) and (38), we obtain the following theorem.

Theorem 5 For $r \in \mathbf{Z}_{+}$, let $p(x) \in \mathbb{P}_{n}(\lambda)$ with

$$
p(x)=\frac{1}{(1-\lambda)^{r}} \sum_{0 \leq k \leq n} C_{k} H_{k}^{(r)}(x \mid \lambda) \quad\left(C_{k} \in \mathbf{Q}(\lambda)\right) .
$$

Then we have

$$
C_{k}=\frac{1}{(1-\lambda)^{r} k !} \sum_{0 \leq j \leq r}\left(\begin{array}{l}
r \\
j
\end{array}\right)(-\lambda)^{r-j} D^{k} p(j)
$$

That is,

$$
p(x)=\frac{1}{(1-\lambda)^{r}} \sum_{0 \leq k \leq n}\left(\sum_{0 \leq j \leq r} \frac{1}{k !}\left(\begin{array}{l}
r \\
j
\end{array}\right)(-\lambda)^{r-j} D^{k} p(j)\right) H_{k}^{(r)}(x \mid \lambda) .
$$

Let us take $p(x)=H_{n}(x \mid \lambda) \in \mathbf{P}_{n}(\lambda)$. Then, by Theorem $5, p(x)=H_{n}(x \mid \lambda)$ can be generated by $\left\{H_{0}^{(r)}(x \mid \lambda), H_{1}^{(r)}(\lambda), \ldots, H_{n}^{(r)}(x \mid \lambda)\right\}$ as follows:

$$
H_{n}(x \mid \lambda)=\sum_{0 \leq k \leq n} C_{k} H_{k}^{(r)}(x \mid \lambda)
$$

where

$$
C_{k}=\frac{1}{(1-\lambda)^{r}} \frac{1}{k !} \sum_{0 \leq j \leq r}\left(\begin{array}{l}
r \\
j
\end{array}\right)(-\lambda)^{r-j} D^{k} p(j)
$$

and

$$
p^{(k)}(x)=D^{k} p(x)=n(n-1) \cdots(n-k+1) H_{n-k}(x \mid \lambda)=\frac{n !}{(n-k) !} H_{n-k}(x \mid \lambda) .
$$


By (40) and (41), we get

$$
C_{k}=\frac{1}{(1-\lambda)^{r}}\left(\begin{array}{l}
n \\
k
\end{array}\right) \sum_{0 \leq j \leq r}\left(\begin{array}{l}
r \\
j
\end{array}\right)(-\lambda)^{r-j} H_{n-k}(j \mid \lambda) .
$$

Therefore, by (39) and (42), we obtain the following theorem.

Theorem 6 For $n \in \mathbf{Z}_{+}$, we have

$$
H_{n}(x \mid \lambda)=\frac{1}{(1-\lambda)^{r}} \sum_{0 \leq k \leq n}\left(\begin{array}{l}
n \\
k
\end{array}\right)\left(\sum_{0 \leq j \leq r}\left(\begin{array}{l}
r \\
j
\end{array}\right)(-\lambda)^{r-j} H_{n-k}(j \mid \lambda)\right) H_{k}^{(r)}(x \mid \lambda) .
$$

Let us assume that $p(x)=H_{n}^{(r)}(x \mid \lambda)$.

Then we have

$$
\begin{aligned}
p^{k}(x) & =n(n-1) \cdots(n-k+1) H_{n-k}^{(r)}(x \mid \lambda) \\
& =\frac{n !}{(n-k) !} H_{n-k}^{(r)}(x \mid \lambda) .
\end{aligned}
$$

From Theorem 1, we note that $p(x)=H_{n}^{(r)}(x \mid \lambda)$ can be expressed as a linear combination of $H_{0}(x \mid \lambda), H_{1}(x \mid \lambda), \ldots, H_{n}(x \mid \lambda)$

$$
H_{n}^{(r)}(x \mid \lambda)=\sum_{0 \leq k \leq n} b_{k} H_{k}(x \mid \lambda)
$$

where

$$
\begin{aligned}
b_{k} & =\frac{1}{(1-\lambda) k !}\left\{p^{k}(1)-\lambda p^{(k)}(0)\right\} \\
& =\frac{n !}{(1-\lambda) k !(n-k) !}\left\{H_{n-k}^{(r)}(1 \mid \lambda)-\lambda H_{n-k}^{(r)}(\lambda)\right\} .
\end{aligned}
$$

By (34) and (45), we get

$$
b_{k}=\left(\begin{array}{l}
n \\
k
\end{array}\right) H_{n-k}^{(r-1)}(\lambda) .
$$

Therefore, by (44) and (46), we obtain the following theorem.

Theorem 7 For $n \in \mathbf{Z}_{+}$, we have

$$
H_{n}^{(r)}(x \mid \lambda)=\sum_{0 \leq k \leq n}\left(\begin{array}{l}
n \\
k
\end{array}\right) H_{n-k}^{(r-1)}(\lambda) H_{k}(x \mid \lambda) .
$$

Remark From (2) and (37), we note that

$$
\begin{aligned}
\frac{d}{d \lambda}\left(\frac{1-\lambda}{e^{t}-\lambda}\right) & =\frac{1-e^{t}}{\left(e^{t}-\lambda\right)^{2}}=\frac{1}{(1-\lambda)^{2}}\left(\frac{(1-\lambda)^{2}}{\left(e^{t}-\lambda\right)^{2}}-\frac{(1-\lambda)^{2}}{\left(e^{t}-\lambda\right)^{2}} e^{t}\right) \\
& =\frac{1}{(1-\lambda)^{2}}\left(\frac{(1-\lambda)^{2}}{\left(e^{t}-\lambda\right)^{2}}-\frac{(1-\lambda)^{2}}{\left(e^{t}-\lambda\right)^{2}}\left(e^{t}-\lambda+\lambda\right)\right)
\end{aligned}
$$




$$
\begin{aligned}
& =\frac{1}{1-\lambda}\left(\frac{(1-\lambda)^{2}}{\left(e^{t}-\lambda\right)^{2}}-\frac{1-\lambda}{e^{t}-\lambda}\right) \\
& =\frac{1}{1-\lambda} \sum_{n=0}^{\infty}\left(H_{n}^{(2)}(\lambda)-H_{n}(\lambda)\right) \frac{t^{n}}{n !},
\end{aligned}
$$

and

$$
\begin{aligned}
\frac{d^{2}}{d \lambda^{2}}\left(\frac{1-\lambda}{e^{t}-\lambda}\right) & =2 ! \frac{1-e^{t}}{\left(e^{t}-\lambda\right)^{3}}=\frac{2 !}{(1-\lambda)^{3}}\left(\frac{(1-\lambda)^{3}}{\left(e^{t}-\lambda\right)^{3}}-\frac{(1-\lambda)^{3}}{\left(e^{t}-\lambda\right)^{3}} e^{t}\right) \\
& =\frac{2 !}{(1-\lambda)^{3}}\left(\frac{(1-\lambda)^{3}}{\left(e^{t}-\lambda\right)^{3}}-\frac{(1-\lambda)^{3}}{\left(e^{t}-\lambda\right)^{3}}\left(e^{t}-\lambda+\lambda\right)\right) \\
& =\frac{2 !}{(1-\lambda)^{2}}\left(\frac{(1-\lambda)^{3}}{\left(e^{t}-\lambda\right)^{3}}-\frac{(1-\lambda)^{2}}{\left(e^{t}-\lambda\right)^{2}}\right) \\
& =\frac{2 !}{(1-\lambda)^{2}} \sum_{n=0}^{\infty}\left(H_{n}^{(3)}(\lambda)-H_{n}^{(2)}(\lambda)\right) \frac{t^{n}}{n !} .
\end{aligned}
$$

Continuing this process, we obtain the following equation:

$$
\begin{aligned}
\frac{d^{k}}{d \lambda^{k}}\left(\frac{1-\lambda}{e^{t}-\lambda}\right) & =\frac{k !}{(1-\lambda)^{k}}\left(\frac{(1-\lambda)^{k+1}}{\left(e^{t}-\lambda\right)^{k+1}}-\frac{(1-\lambda)^{k}}{\left(e^{t}-\lambda\right)^{k}}\right) \\
& =\frac{k !}{(1-\lambda)^{k}} \sum_{n=0}^{\infty}\left(H_{n}^{(k+1)}(\lambda)-H_{n}^{(k)}(\lambda)\right) \frac{t^{n}}{n !} \quad \text { (see [8]). }
\end{aligned}
$$

By (1), (2) and (49), we get

$$
\frac{d^{k}}{d \lambda^{k}} H_{n}(\lambda)=\frac{k !}{(1-\lambda)^{k}}\left(H_{n}^{(k+1)}(\lambda)-H_{n}^{(k)}(\lambda)\right),
$$

where $k$ is a positive integer (see $[7,8])$.

\section{Competing interests}

The authors declare that they have no competing interests.

\section{Authors' contributions}

All authors contributed equally to the manuscript and typed, read, and approved the final manuscript.

\section{Author details}

'Department of Mathematics, Sogang University, Seoul, 121-742, Republic of Korea. ${ }^{2}$ Department of Mathematics, Kwangwoon University, Seoul, 139-701, Republic of Korea.

\section{Acknowledgements}

This research was supported by the Basic Science Research Program through the National Research Foundation of Korea (NRF) funded by the Ministry of Education, Science and Technology 2012R1A1A2003786.

Received: 27 November 2012 Accepted: 7 December 2012 Published: 27 December 2012

\section{References}

1. Araci, S, Acikgoz, M: A note on the Frobenius-Euler numbers and polynomials associated with Bernstein polynomials. Adv. Stud. Contemp. Math. 22(3), 399-406 (2012)

2. Can, M, Cenkci, M, Kurt, V, Simsek, Y: Twisted Dedekind type sums associated with Barnes' type multiple Frobenius-Euler L-functions. Adv. Stud. Contemp. Math. 18(2), 135-160 (2009)

3. Cangul, IN, Kurt, V, Ozden, H, Simsek, Y: On the higher-order w- $q$-Genocchi numbers. Adv. Stud. Contemp. Math. 19(1), 39-57 (2009) 
4. Carlitz, L: A note on q-Eulerian numbers. J. Comb. Theory, Ser. A 25(1), 90-94 (1978)

5. Kim, DS, Dolgy, DV, Kim, T, Rim, S-H: Some formulae for the product of two Bernoulli and Euler polynomials. Abstr. Appl. Anal. 2012, Article ID 784307 (2012)

6. Kim, T, Choi, J: A note on the product of Frobenius-Euler polynomials arising from the $p$-adic integral on $\mathbb{Z}_{p}$. Adv. Stud. Contemp. Math. 22(2), 215-223 (2012)

7. Kim, T: An identity of the symmetry for the Frobenius-Euler polynomials associated with the fermionic $p$-adic invariant $q$-integrals on $\mathbb{Z}_{p}$. Rocky Mt. J. Math. 41(1), 239-247 (2011)

8. Kim, T: Identities involving Frobenius-Euler polynomials arising from non-linear differential equations. J. Number Theory 132(12), 2854-2865 (2012)

9. Kim, T: Symmetry of power sum polynomials and multivariate fermionic $p$-adic invariant integral on $\mathbf{Z}_{p}$. Russ. J. Math. Phys. 16(1), 93-96 (2009)

10. Rim, S-H, Jeong, J: On the modified $q$-Euler numbers of higher order with weight. Adv. Stud. Contemp. Math. 22(1), 93-98 (2012)

11. Rim, S-H, Lee, J: Some identities on the twisted $(h, q)$-Genocchi numbers and polynomials associated with q-Bernstein polynomials. Int. J. Math. Math. Sci. 2011, Article ID 482840 (2011)

12. Ryoo, CS: A note on the Frobenius-Euler polynomials. Proc. Jangjeon Math. Soc. 14(4), 495-501 (2011)

13. Simsek, Y, Bayad, A, Lokesha, V: q-Bernstein polynomials related to $q$-Frobenius-Euler polynomials, I-functions, and q-Stirling numbers. Math. Methods Appl. Sci. 35(8), 877-884 (2012)

14. Simsek, Y, Yurekli, O, Kurt, V: On interpolation functions of the twisted generalized Frobenius-Euler numbers. Adv. Stud. Contemp. Math - Jang'jun Math. Soc. 15(2), 187-194 (2007)

15. Shiratani, K: On the Euler numbers. Mem. Fac. Sci., Kyushu Univ., Ser. A, Math. 27, 1-5 (1973)

16. Shiratani, K, Yamamoto, S: On a p-adic interpolation function for the Euler numbers and its derivatives. Mem. Fac. Sci., Kyushu Univ., Ser. A, Math. 39(1), 113-125 (1985)

doi:10.1186/1029-242X-2012-307

Cite this article as: Kim and Kim: Some new identities of Frobenius-Euler numbers and polynomials. Journal of Inequalities and Applications 2012 2012:307.

\section{Submit your manuscript to a SpringerOpen ${ }^{\ominus}$ journal and benefit from:}

$\checkmark$ Convenient online submission

- Rigorous peer review

Immediate publication on acceptance

Open access: articles freely available online

- High visibility within the field

- Retaining the copyright to your article 